\title{
Proteção Facial e Respiratória: Perspetivas Atuais no Contexto da Pandemia por COVID-19
}

\section{Respiratory and Facial Protection: Current Perspectives in the Context of the COVID-19 Pandemic}

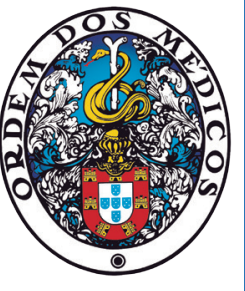

\author{
David PERES $\triangle{ }^{1,2}$, José Pedro BOLÉO-TOMÉ 3 , Gilda SANTOS ${ }^{4}$ \\ Acta Med Port 2020 Sep;33(9):583-592 - https://doi.org/10.20344/amp.14108
}

\section{RESUMO}

A nova pandemia por SARS-CoV-2 é um exemplo de uma emergência de saúde pública de âmbito internacional, associada a consideráveis desafios sociais e económicos. A nível das unidades de saúde há o risco que surtos nosocomiais sejam amplificadores locais. Perante tal, práticas de controlo de infeção são de importância crítica no funcionamento destes serviços, de que faz parte a utilização adequada de equipamento de proteção individual. Este deve ser adequado à via de transmissão do agente que, no caso do SARS-CoV-2, é através de gotícula e contacto. O indivíduo infetado, ao falar, tossir ou espirrar, dissemina gotículas que contêm o vírus, contaminando diretamente outros indivíduos, que estão num raio de um a dois metros, assim como o ambiente. A transmissão por via aérea também poderá ocorrer, no caso de procedimentos geradores de aerossóis. A nível da proteção respiratória existe, atualmente, fraca evidência que a utilização de respiradores permita maior proteção que máscara cirúrgica para o SARS-CoV-2 ou outros vírus (com exceção dos procedimentos geradores de aerossóis, em que a utilização de um respirador é recomendada). A proteção ocular deverá ser garantida sempre que houver risco de salpicos, gotículas ou aerossóis. A utilização incorreta de equipamento de proteção individual, para a via de transmissão do agente ou superior ao necessário, é uma forma de uso indevido e pode afetar o seu suprimento para as situações em que é realmente indicado. A disponibilização deste equipamento de proteção, e formação dos profissionais de saúde na sua correta utilização, é fortemente recomendado para garantir a prestação de cuidados seguros.

Palavras-chave: COVID-19; Dispositivos de Proteção Respiratória; Equipamento de Proteção Individual; Máscaras; SARS-CoV-2

\section{ABSTRACT}

The new SARS-CoV-2 pandemic is an example of an international public health emergency, which is associated with considerable social and economic challenges. At the healthcare level there is the risk that nosocomial outbreaks can be local amplifiers. Adequate infection control practices are of critical importance, which include proper use of personal protective equipment. This equipment must be appropriate to the pathogen transmission route that, in the case of SARS-CoV-2, occurs through droplet and contact routes. The infected individual, when talking, coughing or sneezing, spreads droplets containing the virus, directly contaminating other individuals within one to two meters of distance, as well as the surrounding environment. Airborne transmission may occur when aerosol-generating procedures are performed. Concerning respiratory protection, there is currently weak evidence that the use of respirators provides better protection than surgical masks for SARS-CoV-2 or other viruses (with the exception of aerosol-generating procedures, in which case the use of a respirator is recommended). Eye protection should be guaranteed whenever there is a risk of splashes, droplets or aerosols. The use of different, or higher than necessary, level of personal protective equipment, for the transmission route of the agent, is a form of misuse and can affect its supply for situations when it is clearly indicated. The adequate provision of protective equipment, as well as training of healthcare professionals in its correct use, is highly recommended to ensure safety of care.

Keywords: COVID-19; Masks; Personal Protective Equipment; Respiratory Protective Devices; SARS-CoV-2

\section{INTRODUÇÃO}

A infeção tem um amplo espectro de agentes etiológicos, desde bactérias, vírus, fungos, parasitas ou priões. Várias vias de transmissão estão descritas, nomeadamente via sanguínea, gotículas, aerossóis (também designados de partículas) e contacto (direto e/ou indireto), sendo possível a disseminação por mais do que uma via de transmissão. ${ }^{1}$

A proteção facial e respiratória foca-se na possibilidade da presença de agentes patogénicos no ar, suportados por um "veículo" com um amplo espetro de dimensão. Esta variável determina o número de microrganismos veiculados, a distância que podem percorrer, o grau de penetração no trato respiratório do hospedeiro, bem como o tipo de proteção necessária. Sob o ponto de vista prático, poderá divi- dir-se a dimensão destes 'veículos' em salpicos (dimensão superior a $100 \mu \mathrm{m}$, caem logo após serem emanados para o ar); gotículas (diâmetro entre 5 e $100 \mu \mathrm{m}$, são sujeitos às forças gravitacionais segundos após a sua disseminação no ar) e partículas (inferiores a $5 \mu \mathrm{m}$, com capacidade de se manter suspensas no ar e de percorrer distâncias superiores). ${ }^{2}$

Estes salpicos, gotículas ou partículas são gerados a partir da fonação, espirros, tosse ou resultado de certos procedimentos clínicos. Enquanto a transmissão por salpicos e gotículas tende a ser relativamente eficiente (pois podem veicular grande número de microrganismos, mas numa curta distância), já aquela veiculada por partículas é considerada relativamente ineficiente (sendo necessária proximidade,

1. Unidade de Saúde Pública. Agrupamento de Centros de Saúde do Grande Porto IV - Póvoa de Varzim/ Vila do Conde.. Vila do Conde. Portugal.

2. Comissão de Controlo de Infeção e Resistência aos Antimicrobianos. Unidade Local de Saúde de Matosinhos. Matosinhos. Portugal.

3. Serviço de Pneumologia. Hospital Prof. Doutor Fernando Fonseca. Amadora. Portugal.

4. Departamento de Engenharia de Produto. Centro Tecnológico Têxtil e Vestuário. Vila Nova de Famalicão. Portugal.

$\square$ Autor correspondente: David Peres. david.r.peres@gmail.com

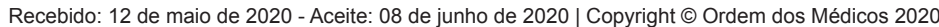


durante períodos de exposição, dispersão e suscetibilidade relevantes), mas com potencial de penetração a nível alveolar. Considerando um espaço fechado, o número de renovações de ar condiciona, diretamente, a diminuição da quantidade de aerossóis. De facto, estima-se que por cada renovação de ar seja reduzida a contaminação em $63 \%$, o que, em termos práticos, ao fim de cinco renovações se pode considerar totalmente descontaminado (desde que a fonte tenha cessado a sua emissão). ${ }^{2}$

Considerando o passado recente, em dezembro de 2019 houve um relato de um conjunto de casos de pneumonia de causa desconhecida em Wuhan, província de Hubei na China, e em janeiro de 2020 a descrição de um novo vírus pertencente à família dos coronavírus. ${ }^{3}$ Estes microrganismos podem causar uma série de doenças em animais e humanos, desde uma coriza até SARS (síndrome respiratória severa aguda) ou MERS (síndrome respiratória do Médio Oriente). ${ }^{4} \mathrm{~A}$ doença associada a este vírus (que se designou SARS-CoV-2) é, atualmente, referida como COVID-19. Perante a rápida disseminação desta doença, no dia 30 de janeiro de 2020 a Organização Mundial da Saúde (OMS) declarou a COVID-19 como uma emergência de saúde pública de âmbito internacional e a 11 de março, uma pandemia. ${ }^{5}$ Esta tem tido grande impacto junto da população, tendo levado à implementação de medidas de distanciamento/ isolamento social, que implicam uma grande mudança de estilo de vida e consequências relevantes a nível económico e social. Simultaneamente, os sistemas de saúde tiveram de se adaptar rapidamente, para responder às crescentes solicitações, inerentes à evolução da situação epidemiológica. A nível das unidades de saúde há o risco de que surtos nosocomiais sejam amplificadores locais importantes. Perante tal, as práticas de controlo de infeção são de importância crítica no funcionamento destas unidades. ${ }^{6}$ Weber et al, ao reverem experiências pandémicas passadas, recomendam que todas as unidades de saúde tenham planos de contingência para lidar com estes agentes patogénicos, nomeadamente a nível de identificação precoce e isolamento de casos, bem como disponibilização de equipamento de proteção individual (EPI) e formação dos profissionais. ${ }^{7}$ De facto, durante o surto de SARS em 2003, houve vários relatos de infeção nosocomial por este agente em profissionais de saúde. ${ }^{8}$ Por sua vez, numa análise de 'lições aprendidas', Cheng et al descrevem que o risco de desenvolver SARS foi 12,6 vezes maior para aqueles profissionais que não usavam máscara cirúrgica durante a prestação de cuidados de saúde. Outros fatores de risco ocupacional foram descritos, tais como: contacto com secreções do trato respiratório, exposição ocular e de membranas mucosas a fluidos corporais, utilização inconsistente de EPI e realização de procedimentos geradores de aerossóis. ${ }^{9} \mathrm{Na}$ atual pandemia estão descritos 3,8\% de casos confirmados em profissionais de saúde chineses, $10 \%$ em Itália e $20 \%$ em Espanha. ${ }^{10}$

No âmbito do quinto componente-chave de atuação do Plano Nacional de Preparação e Resposta à COVID-19 da Direção-Geral da Saúde, definiram-se como objetivos, en- tre outros, fortalecer a segurança dos profissionais, doentes e visitantes das unidades de saúde, assim como a sua capacidade em responder a esta pandemia. ${ }^{11}$ Parte integrante dos planos de contingência é a disponibilização de EPI enquanto pilar importante na prevenção e controlo da transmissão cruzada do SARS-CoV-2, com formação dos profissionais na sua utilização.

Este documento foi estruturado com o intuito de esclarecer e promover as boas práticas na proteção facial e respiratória dos profissionais de saúde, no contexto da atual pandemia por COVID-19. A parte final é dedicada ao panorama nacional de produção deste tipo de equipamentos e suas perspetivas futuras.

\section{MATERIAL E MÉTODOS}

Foi efetuada uma pesquisa da literatura para identificar artigos científicos/ orientações sobre proteção facial e respiratória na prestação de cuidados de saúde, assim como utilização de EPI na COVID-19 e/ou SARS-CoV-2. Os autores identificaram resumos e textos completos da base de dados PubMed até 2 de junho de 2020. Foram ainda consultados documentos técnicos e recomendações/ orientações das seguintes organizações: OMS, Centers for Disease Control and Prevention (CDC), União Europeia (European standards for medical supplies), Public Health England (PHE), European Center for Disease Control and Prevention (ECDC), Direção-Geral da Saúde (DGS), Autoridade para as Condições do Trabalho (ACT) e Autoridade Nacional do Medicamento e Produtos de Saúde (INFARMED). A pesquisa foi restrita para documentos escritos em Inglês e Português.

\section{CARACTERÍSTICAS DAS MÁSCARAS E RESPIRA- DORES}

Uma máscara é um dispositivo médico (DM) que cobre a boca, nariz e queixo, constituindo uma barreira que limita a contaminação por microrganismos, entre o profissional de saúde e o doente. É, também, utilizada pelos profissionais de saúde para impedir que gotículas ou salpicos cheguem à sua boca e nariz, ajudando a reduzir e/ ou controlar a propagação de gotículas da pessoa que usa este dispositivo. ${ }^{12}$ Estudos demonstraram que a mucosa nasal, conjuntiva e, menos frequentemente, a boca, são portas de entrada possíveis a vírus respiratórios. ${ }^{2}$ Siegel et al referem como fatores que influenciam a distância de deslocamento das gotículas a sua velocidade, mecanismo de impulso, densidade das secreções, fatores ambientais (como temperatura e humidade) e a infeciosidade do agente patogénico. Sob o ponto de vista prático, e segundo as precauções de gotícula, preconiza-se a utilização de uma máscara cirúrgica, se o profissional de saúde estiver a menos de um a dois metros do doente. ${ }^{13} \mathrm{~A}$ Norma Europeia 14683, destinada a máscaras faciais com finalidade médica, classifica-as em diferentes tipos (I, II e IIR) segundo as suas características, nomeadamente: a eficiência de filtração bacteriana, a pressão diferencial (respirabilidade), a resistência aos salpicos e a carga microbiológica. ${ }^{14}$ 
Podem ser munidas de atilhos ou elásticos e ter, ou não, incorporada uma viseira (ver Tabela 1).

Um respirador (também designado por 'semi-máscara de proteção respiratória' ou 'aparelho de proteção respiratória filtrante') é um tipo de EPI, tendo como principal função proteger quem o utiliza de ser contaminado com partículas emanadas através de espirros, tosse ou resultado de procedimentos geradores de aerossóis (PGA). ${ }^{2}$ As partículas têm a capacidade de se manter suspensas no ar e, como tal, devem ser instituídas as precauções de partícula (ou via aérea), que incluem a utilização de um respirador por todos aqueles presentes no mesmo espaço que a fonte de transmissão. ${ }^{13}$

A nível normativo, os EPI devem estampar a marcação CE, que indica que os mesmos cumprem os requisitos harmonizados e podem ser comercializados na União Europeia. ${ }^{15}$ Os respiradores deverão também obedecer à Norma Europeia 149, segundo a qual são divididos em três classes, nomeadamente: FFP1, FFP2 e FFP3 (ver Tabela 1). Por sua vez, nos Estados Unidos da América (EUA), o National Institute of Ocupational Safety and Health (NIOSH) classifica os respiradores em N (non oil resistant), R (oil resistant) e $\mathrm{P}$ (oil proof). Nos primeiros, é feita uma divisão segundo a eficácia de filtração de partículas em 95\%, 99\% e $99,97 \% .^{16}$ Em termos práticos, um respirador N95 é equivalente a um respirador FFP2 europeu (ou ao KN95 chinês) e tem uma capacidade de filtração de partículas igual ou superior a 95\%. Vários estudos comparam a eficácia das máscaras cirúrgicas versus respiradores na prevenção da transmissão cruzada do vírus influenza: os resultados de uma meta-análise, publicada em 2017, apoiam a utilização do respirador. No entanto, a evidência encontrada foi fraca e os resultados, entre os estudos, foram inconsistentes. ${ }^{17}$ Já dois outros estudos mais recentes (uma meta-análise e um ensaio clínico randomizado) não encontraram diferença, estatisticamente significativa, na utilização do respirador, quando comparado com a máscara cirúrgica. ${ }^{18,19}$ Por sua vez, em junho de 2020, uma revisão sistemática e meta-análise envolvendo 172 estudos observacionais concluiu que a utilização de máscara permite uma grande redução no risco de infeção por COVID-19 (aOR 0,15; IC 95\% 0,07 - 0,34), assim como a utilização de proteção ocular (aOR 0,22; IC 95\% 0,12 - 0,39). Sugeriu, embora com pouca certeza, que a utilização de respirador poderá permitir uma proteção adicional (versus máscara cirúrgica). ${ }^{20}$

A nível europeu, o Regulamento (EU) 2016/425 define os requisitos de conceção e fabrico dos EPI. Por sua vez, para os DM, a sua definição consta na Diretiva 93/42/ CEER e Regulamento (UE) 2017/745. Recentemente, com o objetivo de assegurar a disponibilidade de EPI e DM para a pandemia por COVID-19, foi emanada a recomendação (UE) $2020 / 403^{21}$ e, a nível nacional, o Decreto-Lei n 14 $\mathrm{E} / 2020 .^{22}$

Por sua vez, a proteção ocular (óculos ou viseiras) deverá cumprir as especificações da Norma Europeia 166 (Tabela 1). Segundo a NIOSH, a utilização deste tipo de

Tabela 1 - Caraterísticas técnicas das máscaras, respiradores e proteção ocular, segundo o enquadramento normativo europeu

\section{MÁSCARAS CIRÚRGICAS (EN 14683)}

Funções: protege da disseminação e/ou inalação de gotículas (> $5 \mu \mathrm{m})$

$\begin{array}{lccc}\text { Tipo de máscara } & \text { I } & \text { II } & \text { IIR } \\ \text { Eficácia filtração bacteriana }(\%) & \geq 95 & \geq 98 & \geq 98 \\ \text { Respirabilidade - Delta } \mathrm{P}(\mathrm{Pa}) & <24,9 & <24,9 & <49,0 \\ \text { Resistência à passagem sangue }(\mathrm{mmHg}) & - & - & >120\end{array}$

\section{RESPIRADORES (EN 149)}

Função: protege da inalação de partículas $(<5 \mu \mathrm{m})$

\section{Classe de respirador}

Fuga total para o interior (\%)

Retenção total (\%)

Penetração no filtro (\%)

Eficácia de fltração de partículas (\%)

Máxima resistência respiratória (mBar)

$\begin{array}{ccc}\text { FFP1 } & \text { FFP2 } & \text { FFP3 } \\ 22 & 8 & 2 \\ 78 & 92 & 98 \\ 20 & 6 & 1 \\ \geq 80 & \geq 94 & \geq 99 \\ 2,1 & 2,4 & 3\end{array}$
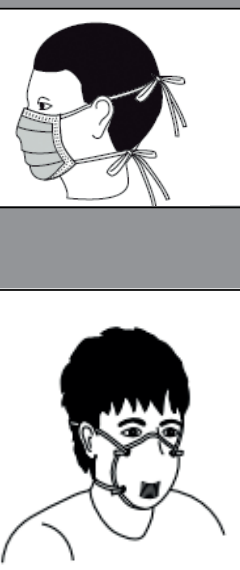

\section{PROTEÇÃO OCULAR (EN 166)}

\section{Óculos de proteção ou viseiras}

$\begin{array}{ll}\text { Classe ótica } & 1,2 \text { ou } 3 \\ \text { Proteção partículas em alta velocidade } & \mathrm{A}(\mathrm{T}) \text { - alta } \\ \mathrm{B}(\mathrm{T}) \text { - média } & \mathrm{F}(\mathrm{T}) \text { - baixa } \\ \mathrm{S}-\text { - mínima } \\ 7 \text { tipos }\end{array}$

Fonte das imagens: Center for Disease Control and Prevention e Public Health England 
equipamento deverá ser confortável, permitindo um bom ajuste facial e visão periférica sem embaciar, assim como assegurar compatibilidade com a máscara ou respirador. De ressalvar que óculos de correção ou lentes de contacto não são considerados meios de proteção. ${ }^{13}$

Com o intuito de prevenir a transmissão cruzada proveniente de fontes de infeção conhecidas (ou não) e de modo a garantir a segurança dos utentes e profissionais de saúde, as precauções básicas em controlo de infeção devem ser aplicadas quotidianamente. Segundo estas, deverá ser considerada a utilização de proteção facial e/ou respiratória sempre que houver risco de projeção de salpicos de fluidos orgânicos para a face e durante os PGA's. ${ }^{23}$

Existe ainda outro tipo de proteção conhecido como 'máscaras comunitárias' (ou 'máscaras para uso social') que, segundo o ECDC, incluem várias formas de máscaras de fabrico 'caseiro' ou industrial, feitas de tecido ou de outros têxteis ou materiais, como o papel. Estes equipamentos não são padronizados e não se destinam a ser utilizados no âmbito da prestação de cuidados de saúde. ${ }^{12}$ Este relatório técnico do ECDC conclui que, no âmbito da atual pandemia por COVID-19, a sua utilização pela população, especialmente em locais fechados e movimentados, pode reduzir a disseminação da infeção, na comunidade, através da contenção de gotículas de indivíduos infetados que ainda não desenvolveram sintomas ou que permanecem assintomáticos. Alerta-se, no entanto, para o risco de constituir uma falsa sensação de segurança (levando a que outras medidas recomendadas não sejam adequadamente cumpridas), assim como a possibilidade da própria máscara, quando mal utilizada, poder aumentar o risco de transmissão (mãos tocam na máscara contaminada, não são higienizadas, contaminando superfícies e face do utilizador). Por sua vez, a DGS refere que "aplicando-se o princípio da precaução em Saúde Pública, é de considerar o uso de máscaras por todas as pessoas que permaneçam em espaços interiores fechados com múltiplas pessoas, como medida de proteção adicional ao distanciamento social, à higiene das mãos e à etiqueta respiratória". ${ }^{24}$

\section{BOAS PRÁTICAS NA UTILIZAÇÃO DE MÁSCARAS E RESPIRADORES}

A utilização adequada destes dispositivos/equipamentos é imprescindível para que cumpram a sua função. Neste sentido, tanto a OMS como o ECDC, e a sua congénere norte-americana $\mathrm{CDC}$, tecem recomendações nesta área, as quais se resume de seguida. . $^{25-27}$

Para colocação, deverá ter-se em atenção os seguintes passos:

1. Higienizar as mãos (com água e sabão ou uma solução antissética de base alcoólica - SABA);

2. Colocar a máscara, cobrindo a boca, nariz e queixo;

3. Prender à cabeça com os atilhos (dando um laço em cada um) ou com os elásticos, sem cruzar;

4. Ajustar a banda flexível na zona do nariz;

5. Certificar que a máscara está bem ajustada à face;

6. Em caso de utilização de proteção ocular, esta de- verá ser colocada após a máscara.

Em relação ao respirador, é vital confirmar que não existem fugas entre o equipamento e a face, as quais podem ser verificadas através de um simples teste: com o respirador colocado, inspirar vigorosamente. Se não existirem fugas, o respirador deverá colapsar. Ao expirar poder-se-á também verificar se há fugas nas zonas de contacto do respirador com a face. Existe, ainda, um teste qualitativo para ajuste dos respiradores, devidamente descrito num documento técnico do ECDC. ${ }^{28}$ De referir que a existência de pilosidade facial (e respetiva forma e densidade) pode afetar a eficácia do respirador. Dadas estas particularidades, a NIOSH alerta que os potenciais utilizadores de respiradores devem ter formação prévia sobre este tipo de EPI. ${ }^{29}$

Enquanto a máscara/respirador estiver colocada/o, deverá atender-se a:

1. Evitar tocar na máscara/respirador com as mãos. Se tal acontecer, higienizar as mãos (com água e sabão ou SABA);

2. Substituir a máscara/ respirador, se estiver danificada/o, húmida/o, visivelmente contaminada/o ou no caso de se tornar difícil respirar;

3. Tanto as máscaras como os respiradores estão projetados para 8 horas de utilização ininterrupta. No entanto, na prática, é recomendado um tempo máximo até 4 a 6 horas ${ }^{6,30}$;

4. Se a máscara/ respirador for retirada/o, deverá ser rejeitada/o, as mãos higienizadas e colocada/o um novo dispositivo/ equipamento.

Por fim, recomenda-se o seguinte procedimento para a sua remoção:

1. No caso de proteção ocular colocada, deverá ser retirada antes da máscara/respirador, tendo o cuidado de:

a. Não tocar na sua parte frontal (risco de contaminação);

b. Se for de uso único, rejeitar para resíduos Grupo III - risco biológico;

c. Se for de uso múltiplo, colocar em local seguro para descontaminação (por ex: contentor).

2. Retirar, de seguida, a máscara/respirador, não tocando na sua parte frontal:

a. Se se tratar de máscara com atilhos: desapertar o atilho de baixo e depois o de cima;

b. Se for respirador: levantar os elásticos, um de cada vez.

3. Retirar da face, tocando somente nos atilhos/elásticos;

4. Rejeitar para resíduos Grupo III - risco biológico;

5. Após rejeitar, higienizar as mãos (com água e sabão ou uma SABA).

\section{PROTEÇÃO FACIAL E RESPIRATÓRIA NA COVID-19}

A nova pandemia por SARS-CoV-2 é uma emergência de saúde pública cujo cenário epidemiológico está em constante mutação. Perante a presente incerteza relativamente aos mecanismos associados à sua transmissibilidade, 
tanto a OMS, como o ECDC e CDC consideram como via de transmissão do SARS-CoV-2 a gotícula e contacto. , $^{, 31,32}$ $O$ indivíduo infetado, ao falar, tossir ou espirrar, dissemina gotículas que contêm o SARS-CoV-2, contaminando diretamente outros indivíduos que estão num raio de um a dois metros, assim como o ambiente. A transmissão por contacto resulta da capacidade do vírus em se manter viável, nas superfícies, por um período de horas ou até dias. Há, assim, o risco de um indivíduo se infetar ao tocar nessas superfícies e, posteriormente, na face (via contacto indireto). De facto, uma revisão recente analisa 22 estudos publicados que revelam que os coronavírus podem persistir em superfícies inanimadas como metal, vidro ou plástico até nove dias. ${ }^{33}$ Por outro lado, de acordo com van Doremalen et al, a estabilidade ambiental do SARS-CoV-2 é de até três horas pós-aerossolização, até quatro horas no cobre, até 24 horas em cartão e até três dias em plástico e aço inoxidável. Os autores alertam, no entanto, que estes estudos experimentais podem não traduzir cabalmente o comportamento real dos fómites na transmissão da infeção. ${ }^{34}$

A grande maioria dos autores refere que, segundo a atual evidência científica, o coronavírus não é transmitido por via aérea (o que implicaria a aplicação de precauções de partícula, com a utilização de um respirador pelo profissional de saúde), com a exceção da situação em que se realizam PGA's. ${ }^{35}$ Se por um lado, Liu et al, ao estudarem dois surtos por SARS-CoV-2, relatam a possibilidade de aerossolização (encontrando níveis muitos baixos nas enfermarias, mas altos nas instalações sanitárias dos doentes), ${ }^{36}$ por outro lado, Wong et al, num setting semelhante, encontram resultados não compatíveis com transmissão por via aérea, sugerindo que a máscara cirúrgica é proteção respiratória suficiente para os profissionais. ${ }^{37}$ Rubens et al, num comentário ao estudo de van Doremalen et al, referem ainda outros dois estudos, relativos ao SARS-CoV-1, onde não se encontrou este vírus em amostras do ar ambiente provenientes de quartos de doentes infetados e, um outro, com achados semelhantes, referente ao SARS-CoV-2. ${ }^{38}$

Numa comunicação de 19 de março de 2020, a OMS recomendou a utilização das precauções por gotícula e contacto e, no caso de PGA's, precauções de partícula. ${ }^{31}$ Tal orientação é seguida pela maioria dos países, inclusivamente Portugal. ${ }^{30}$ Atualmente considera-se, pois, que o risco de transmissão da infeção por SARS-CoV-2 seja decorrente das vias de gotícula e contacto, e não da transmissão por partícula (com a exceção de PGA's) (Fig. 1).

De referir ainda que, com base numa revisão recente da Cochrane, a evidência para a utilização de um tipo de equipamento (por ex: respiradores FFP3/ FFP2/ N95) em detrimento de outro (por exemplo, máscara cirúrgica) não é tão robusta quanto se poderia considerar, com uma falta de evidência clara de benefício dos respiradores. ${ }^{39}$ É importante lembrar que o EPI é apenas uma parte de um sistema para evitar a contaminação, de que fazem parte outras medidas, nomeadamente: adesão rigorosa à higiene das mãos; criação de circuitos distintos para casos suspeitos/ confirmados de COVID-19; reforço da descontaminação de equipamento e superfícies; boas práticas e formação na colocação e remoção do EPI (o risco de contaminação do profissional é elevado na remoção inadequada do EPI); descontaminação de equipamento de uso múltiplo entre utilizações e triagem/ acondicionamento de resíduos. ${ }^{35}$

Cook alerta que a utilização incorreta ou superior ao necessário de EPI (para a via de transmissão do agente) é uma forma de uso indevido e pode significar suprimento inadequado no futuro. ${ }^{35}$ De facto, tal como Livingston et al

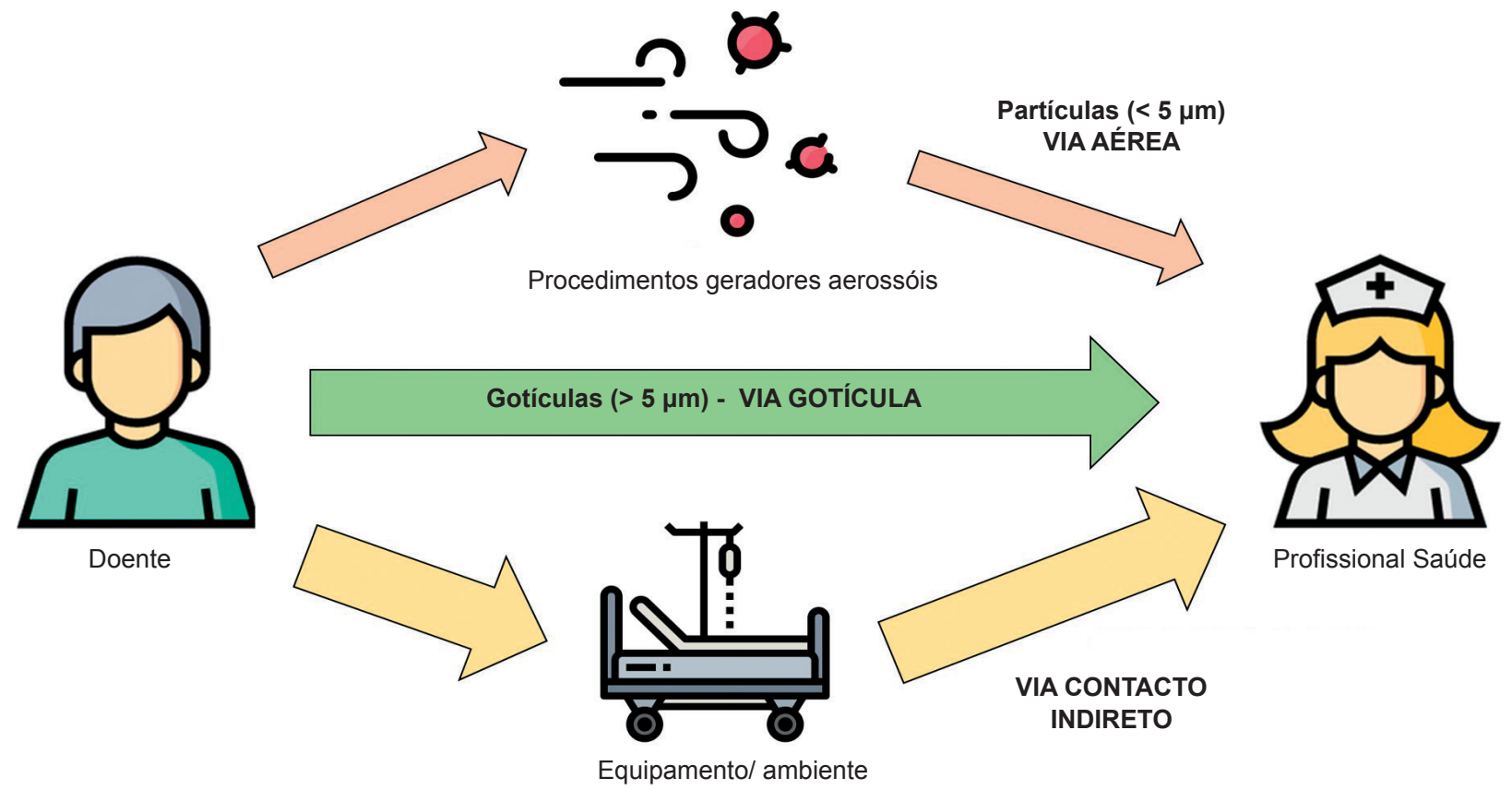

Figura 1 - Vias de transmissão possíveis do SARS-CoV-2

Fonte dos ícones: www.flaticon.com 
referem, o EPI, anteriormente ubiquitário e disponível no ambiente da prestação de cuidados de saúde, é hoje um bem escasso e precioso em muitos locais onde se prestam cuidados a doentes altamente infeciosos. ${ }^{40} \mathrm{Na}$ otimização da sua disponibilidade, a OMS definiu três estratégias a implementar: utilização judiciosa, minimização da necessidade e coordenação da cadeia de fornecimento. ${ }^{41}$ Para auxiliar no planeamento das necessidades, o ECDC publicou um relatório técnico que estima as necessidades de EPI no tratamento de doentes com COVID-19.42 Perante a possibilidade de rutura de stock é recomendando que profissionais sejam alocados a realizar determinada tarefa, executando um conjunto de procedimentos com o mesmo EPI num determinado local (por ex: colheita de amostras para diagnóstico). Também a concentração de casos de COVID-19 em áreas/ serviços específicos permite maximização de recursos, a vários níveis. ${ }^{6}$ Ferioli et al referem, ainda, a possibilidade da telemedicina ou utilização de barreiras físicas em zonas de atendimento administrativo/ triagem. ${ }^{43} \mathrm{Em}$ relação à proteção respiratória e facial, o ECDC estabelece priorização destes dispositivos/ equipamentos. Assim, a nível dos respiradores, são os profissionais de saúde que têm prioridade na sua utilização (principalmente aqueles que realizam PGA's), salvaguardando que estes poderão ser utilizados na abordagem a vários doentes, a menos que estejam danificados, visivelmente contaminados ou húmidos. $\mathrm{Na}$ ausência de respiradores, os profissionais de saúde devem usar máscaras com a mais alta eficácia de filtração disponível. ${ }^{6}$

A nível das máscaras cirúrgicas (e no caso de não exis- tirem respiradores disponíveis), a prioridade para a sua utilização são os cuidadores de doentes com COVID-19, seguido dos casos confirmados sintomáticos de COVID-19 e, por fim, dos casos suspeitos. ${ }^{6}$

A Tabela 2 pretende sistematizar a proteção facial e/ou respiratória necessária em contexto da pandemia por $\mathrm{CO}$ VID-19, considerando a prestação de cuidados de saúde a menos de dois metros de distância do doente, tendo como variáveis doentes não suspeitos versus doentes suspeitos/ confirmados, zonas de isolamento e realização de PGA's. Tal informação foi estruturada tendo por base orientações da OMS, CDC, ECDC, PHE e DGS e pretende ser meramente orientadora, sem ser exaustiva. $6,27,30,41,44$

Considerando a existência de transmissão comunitária de SARS-CoV-2, tanto a DGS como a PHE recomendam a utilização de uma máscara cirúrgica pelos profissionais de saúde sempre que prestem cuidados a doentes em que não há suspeita de serem COVID-19 positivo (segundo a PHE, esta deverá ser resistente a fluidos, isto é, tipo IIR). ${ }^{30,44}$ No caso de doentes suspeitos ou confirmados (fora de zonas de isolamento e sem PGA's) também deverá ser utilizada uma máscara cirúrgica (segundo a PHE, tipo IIR), assim como proteção ocular. Nestas situações, a DGS refere que se poderá utilizar, em alternativa à máscara, um respirador FFP1 ou, se o doente não tiver máscara colocada, um respirador FFP2. ${ }^{30}$ Por sua vez, todas as recomendações consultadas apontam para a necessidade de utilizar respirador FFP2 (ou FFP3, se alto risco) e proteção ocular ao entrar em zonas de isolamento e/ou realizar PGA's a casos suspeitos ou confirmados de COVID-19.6,27,30,41,44 Nestas

Tabela 2 - Orientação para proteção facial e respiratória na prestação de cuidados de saúde, numa distância inferior a dois metros entre profissional e doente, em contexto de pandemia por COVID-19 (baseado em ECDC ${ }^{6}, \mathrm{CDC}^{27}$, DGS ${ }^{30}, \mathrm{OMS}^{41}$ e PHE $^{44}$ )

\begin{tabular}{|c|c|c|c|c|c|c|c|c|}
\hline \multirow{2}{*}{$\begin{array}{l}\text { Status do } \\
\text { doente }\end{array}$} & \multirow{2}{*}{ Prestação Cuidados Saúde } & \multicolumn{3}{|c|}{ Máscara cirúrgica } & \multicolumn{3}{|c|}{ Respirador } & \multirow{2}{*}{$\begin{array}{l}\text { Proteção } \\
\text { Ocular }\end{array}$} \\
\hline & & I & II & IIR & FFP1 & FFP2 & FFP3 & \\
\hline \multirow{3}{*}{ 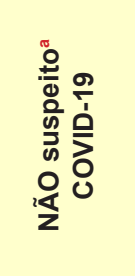 } & $\begin{array}{l}\text { Prof. Saúde: com ou sem } \\
\text { contacto direto }\end{array}$ & & $\begin{array}{l}\checkmark \\
30\end{array}$ & $\begin{array}{c}\checkmark \\
30,44\end{array}$ & & & & $\begin{array}{l}\text { segundo risco } \\
\text { percecionado }\end{array}$ \\
\hline & $\begin{array}{l}\text { Prof. Saúde: } \\
\text { com contacto direto e PGA }\end{array}$ & & & & & $\begin{array}{c}\checkmark \\
30,44\end{array}$ & & $\begin{array}{c}\checkmark \\
30,44\end{array}$ \\
\hline & $\begin{array}{l}\text { Doente } \\
\text { (se colaborante e/ou tolerar) }\end{array}$ & $\begin{array}{c}\checkmark \\
6,27,30,41,44\end{array}$ & & & & & & \\
\hline \multirow{3}{*}{ 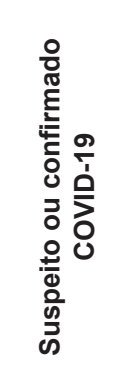 } & $\begin{array}{l}\text { Prof. Saúde: sem PGA } \underline{e} \\
\text { fora de zonas isolamento }\end{array}$ & & $\begin{array}{c}\checkmark \\
6,27,30,41\end{array}$ & $\begin{array}{c}\checkmark \\
6,27,30,41,44\end{array}$ & $\begin{array}{l}\text { alternativa } \\
\text { à máscara } \\
\text { cirúrgica }^{30}\end{array}$ & $\begin{array}{c}\text { alternativa, } \\
\text { se doente } \\
\text { sem } \\
\text { máscara }^{30}\end{array}$ & & $\begin{array}{c}\checkmark \\
6,27,30,41,44\end{array}$ \\
\hline & $\begin{array}{l}\text { Prof. Saúde: com PGA e/ou } \\
\text { dentro de zonas isolamento }\end{array}$ & & & & & $\begin{array}{c}\checkmark \\
6,27,30,41,44\end{array}$ & $\begin{array}{c}\checkmark \\
\text { se alto risco } \\
6,27,30,41,44\end{array}$ & $\begin{array}{c}\checkmark \\
6,27,30,41,44\end{array}$ \\
\hline & $\begin{array}{l}\text { Doente } \\
\text { (se colaborante e/ou tolerar) }\end{array}$ & $\begin{array}{c}\checkmark \\
6,27,30,41,44\end{array}$ & $\begin{array}{c}\checkmark \\
6,27,30,41,44\end{array}$ & & & & & \\
\hline
\end{tabular}

Prof. Saúde: profissional de saúde. PGA: procedimento gerador de aerossóis

(a) Doente não suspeito: aquele que tem teste negativo e/ou cujo inquérito epidemiológico para fatores de risco foi negativo (ver Apêndice 1: https://www.actamedicaportuguesa.com/ revista/index.php/amp/article/view/14108/Apendice_01.pdf).

(b) Zonas de isolamento: quarto (isolamento estrito) ou enfermaria (isolamento em coorte) ou zonas ADC (áreas dedicadas ao COVID-19) hospitalares e da comunidade ou interior de célula sanitária de ambulância transporte de casos suspeitos ou confirmados de COVID-19.

Nota: o ECDC e CDC recomendam a utilização de respirador em todos os casos suspeitos ou confirmados de COVID-19, no entanto, consideram como alternativa aceitável, uma máscara cirúrgica, desde que não envolva PGA (ver Apêndice 2: https://www.actamedicaportuguesa.com/revista/index.php/amp/article/view/14108/Apendice_02.pdf). 
situações, perante a indisponibilidade de respirador, o profissional poderá utilizar uma máscara cirúrgica (idealmente, tipo IIR), sempre com proteção ocular (idealmente, viseira). ${ }^{6,27}$ Em ambiente de prestação de cuidados de saúde e sempre que a situação clínica o permitir, os doentes deverão utilizar máscara cirúrgica. $6,27,30,41,44$

\section{A PARTICULARIDADE DOS PROCEDIMENTOS GE- RADORES DE AEROSSÓIS}

Grande parte do conhecimento sobre o risco associado a PGA's provém de estudos realizados previamente à pandemia por SARS-CoV-2. Este tipo de procedimento coloca os profissionais de saúde em alto risco de exposição a agentes infeciosos por via aérea, sobretudo se não for utilizado EPI adequado. ${ }^{43}$

Dados relativos a surtos prévios de SARS-CoV-1, MERS e influenza A indicam um risco aumentado de transmissão associado a determinados procedimentos, sendo a entubação endotraqueal aquele que consistentemente apresenta o risco maior. Em relação a este procedimento, uma revisão de Tran et al, publicada em 2012, encontrou um odds ratio combinado de vários estudos de 6,6. ${ }^{45}$ No entanto, noutros, como aspiração de secreções, broncoscopia, administração de medicação nebulizada, uso de máscaras de oxigénio, oxigenoterapia de alto fluxo por cânula nasal (HFNC), desfibrilhação ou compressões torácicas, entre outros, não foram encontradas associações consistentes para transmissão cruzada. ${ }^{45} \mathrm{~A}$ geração de aerossóis ocorre através de um de dois mecanismos: procedimen- tos que criam e dispersam mecanicamente aerossóis (por ex: entubação ou broncoscopia) e técnicas que induzem o doente a produzir e emitir aerossóis (por ex: ventilação por máscara).$^{46} \mathrm{~A}$ evidência existente sobre a transmissão por via aérea foi essencialmente obtida a nível laboratorial, em condições controladas, com simuladores em ambiente de pressão negativa. ${ }^{43}$ Apesar de nem sempre existir evidência consistente, vários autores e organismos propõem listas de PGA's com risco de transmissão por via aérea alguns dos quais descritos na Tabela 3 .

Em manobras de reanimação, além da entubação traqueal, outros procedimentos podem ter risco elevado. No entanto, é difícil quantificá-los individualmente, uma vez que em geral os mesmos profissionais estão envolvidos neste conjunto de procedimentos, que ocorrem de forma sequencial. ${ }^{48}$ Têm sido propostas várias estratégias para minimizar o risco na entubação, nomeadamente a sua realização em ambiente com pressão negativa, utilizando sequência rápida, com um número restrito de profissionais e bem diferenciados. ${ }^{49}$

A broncoscopia também está associada a um risco aumentado de aerossolização, tal como documentado durante a pandemia de influenza A. ${ }^{50}$ Nesse sentido, as principais Sociedades das Especialidades Médicas emitiram recomendações sobre as indicações e cuidados a ter durante estas técnicas que devem, sempre que possível, ser evitadas em doentes COVID-19. .1,52 $^{2}$

Outras questões importantes têm sido levantadas pela ventilação não-invasiva (VNI). Depois dos primeiros relatos

Tabela 3 - Lista de procedimentos geradores de aerossóis, segundo evidência de risco aumentado de transmissão cruzada por via aérea (adaptado de Health Protection Surveillance Centre) $)^{47}$

\begin{tabular}{|c|c|}
\hline Evidência de risco aumentado de transmissão & Procedimentos geradores de aerossóis (PGA's) \\
\hline $\begin{array}{l}\text { RISCO RECONHECIDO } \\
\text { (reconhecido de forma consistente) }\end{array}$ & $\begin{array}{l}\text { - Entubação/ extubação traqueal; } \\
\text { - Traqueostomia, cricotomia; } \\
\text { - } \text { Broncoscopia; } \\
\text { - } \text { Ventilação manual pré-entubação; } \\
\text { - } \quad \text { Expetoraçação induzida. } \\
\text { Associado a dispositivos rotatórios de alta velocidade: } \\
\text { - Em Medicina Dentária: aparelhos de ultrassons, utilização de turbina, } \\
\text { polimento com jato de partículas; } \\
\text { - Certos procedimentos cirúrgicos (por ex: neurocirurgia, cirurgia maxilo- } \\
\text { - facial, otorrinolaringologia, etc.); }\end{array}$ \\
\hline $\begin{array}{l}\text { RISCO PROVÁVEL } \\
\text { (geralmente reconhecido) }\end{array}$ & $\begin{array}{l}\text { - Ventilação não-invasiva (VNI) - CPAP/ BiPAP; } \\
\text { - Oxigenoterapia de alto fluxo por cânula nasal (HFNC). }\end{array}$ \\
\hline $\begin{array}{l}\text { RISCO PLAUSÍVEL } \\
\text { (sem evidência) }\end{array}$ & $\begin{array}{l}\text { - Laringoscopia; } \\
\text { - Endoscopia digestiva alta; } \\
\text { - Ecografia transesofágica; } \\
\text { - Estudo endoscópico da deglutição. }\end{array}$ \\
\hline $\begin{array}{l}\text { RISCO POUCO PROVÁVEL } \\
\text { (sem evidência ou plausibilidade; não reconhecido) }\end{array}$ & $\begin{array}{l}\text { - } \quad \text { Colheita por zaragatoa naso- ou orofaríngea; } \\
\text { - } \quad \text { Aspiração de secreções através de sistema fechado; } \\
\text { - } \quad \text { Adrodução de sonda nasogástrica; } \\
\text { - } \quad \text { Cinesiterap̧ãa respiratória (e na ausência de outros PGA's); } \\
\text { - Endoscopia digestiva baixa. }\end{array}$ \\
\hline
\end{tabular}


terem descrito uma elevada taxa de insucesso com estas técnicas na COVID-19, outros, mais recentes, têm referido que podem ser uma importante arma terapêutica no tratamento destes doentes. Este procedimento pode evitar entubações, sobretudo numa altura em que a escassez de vagas em cuidados intensivos é uma realidade em muitos países. De referir que experiências passadas com o SARS relatam ausência de aumento de risco de transmissão do vírus com a utilização de VNI (desde que utilizado EPI adequado). ${ }^{53} \mathrm{~A}$ utilização de pressão positiva contínua nas vias aéreas (CPAP), através de máscara oronasal ou de helmet bem ajustados, minimizam a contaminação aérea, sobretudo se for realizada em ambiente com pressão negativa. ${ }^{42}$ A própria OMS reconheceu a utilidade da VNI em doentes COVID-19, desde que seja usado EPI adequado. O uso de máscaras de VNI não ventiladas, com colocação de circuitos duplos e uso de filtros nos pontos de entrada e saída, são também recomendados. ${ }^{31,54} \mathrm{Ng}$ et al relatam a exposição de 35 profissionais de saúde, utilizando uma máscara cirúrgica (e não um respirador), aos PGA's através de VNI, intubação e extubação num doente COVID-19 positivo. Curiosamente nenhum destes profissionais desenvolveu doença, tendo tido todos os testes de follow-up negativos para SARS-CoV-2. ${ }^{55}$

$A$ administração de $\mathrm{O}_{2}$ por máscara, ou através de HFNC, também envolve um risco de aerossolização, mas inferior ao da VNI e dependente do fluxo utilizado. Estes riscos podem ser reduzidos através da utilização de $\mathrm{O}_{2}$ por máscara sem humidificação e evitando os nebulizadores em aerossol. ${ }^{43}$

A nível cirúrgico devem considerar-se como passíveis de ser PGA's aqueles associados a dispositivos rotatórios de alta velocidade, como acontece, por exemplo, em certos procedimentos de neurocirurgia, cirurgia maxilo-facial, otorrinolaringologia ou outros. ${ }^{44,47,56}$ Segundo o Intercollegiate General Surgery Guidance do Reino Unido, considera-se que a laparoscopia apresenta, também, algum risco de formação de aerossóis, recomendando-se precaução na sua realização. ${ }^{57}$

\section{INOVAÇÃO NACIONAL NA PRODUÇÃO DE EQUIPA- MENTOS DE PROTEÇÃO}

Nesta parte final pretende-se dar a conhecer o que tem sido feito a nível nacional na produção e certificação de máscaras de utilização comunitária. De facto, a indústria têxtil e do vestuário em Portugal está a enfrentar novos desafios, reunindo esforços para mitigar o impacto da pandemia através do desenvolvimento de EPI e DM. Para tal, foram definidas orientações para a sua colocação no mercado sem marcação CE (regime excecional e transitório), por parte de fabricantes nacionais que habitualmente não os produzia. ${ }^{22}$ Num comunicado conjunto da DGS, INFARMED, Autoridade de Segurança Alimentar e Económica (ASAE), Instituto Português da Qualidade (IPQ) e Centro Tecnológico Têxtil e do Vestuário de Portugal (CITEVE), de 14 de abril de 2020, foi implementado um sistema de categorização de máscaras a ser utilizadas na atual pande- mia, com definição de três níveis: (1) profissionais de saúde e doentes (máscaras tipo II ou IIR); (2) profissionais em contacto frequente com o público (máscaras tipo I ou, em alternativa, máscaras têxteis de uso único ou reutilizáveis, com desempenho mínimo de filtração de 90\%) e (3) profissionais que não estejam em teletrabalho ou população em geral para as saídas autorizadas em contexto de confinamento (máscaras têxteis de uso único ou reutilizáveis, com desempenho mínimo de filtração de $70 \%$ ). O referido comunicado refere, ainda, que foram definidos critérios e requisitos a cumprir pelas máscaras de uso comunitário (classificadas como artigos têxteis), para que seja possível a sua produção nacional. ${ }^{58}$

Um dos principais desafios no desenvolvimento concreto destes equipamentos é a seleção das matérias-primas, particularmente para máscaras, onde existe a necessidade de garantir as propriedades de retenção de partículas e respirabilidade, e onde normalmente se usam tecidos-não-tecidos. Dada a sua escassez no mercado, tem sido necessário analisar diversos substratos têxteis e proceder ao estudo e análise de diferentes combinações, com o objetivo de encontrar soluções para a produção de máscaras descartáveis e/ou reutilizáveis. Além dos requisitos de desempenho é, também, necessário conjugar os fatores de usabilidade, limpeza e descontaminação e conforto.

Não obstante os desafios encontrados, os resultados obtidos até ao momento são bastante positivos, tendo o CITEVE recebido já centenas de protótipos e pedidos de certificação das empresas nacionais. As listas dos materiais e dos equipamentos aprovados encontram-se em atualização constante no website do CITEVE, salientando-se a existência de mais de 150 máscaras aprovadas. ${ }^{59} \mathrm{De}$ referir, ainda, que foi lançado o selo "Máscaras - COVID-19 Aprovado" que permite a consumidores e produtores reconhecer máscaras ou matérias-primas que foram testadas e validadas por uma entidade independente, com laboratórios acreditados. Este elemento distintivo informa, também, se se trata de uma máscara de uso único ou se é reutilizável e, neste caso, a indicação do número de vezes que poderá ser lavada sem afetar o seu desempenho, bem como qual o tipo de utilização para que foi aprovada: uso profissional ou uso geral. Finalmente, o selo apresenta ainda um $Q R$ Code de acesso ao website onde os consumidores poderão ver a lista de máscaras aprovadas. ${ }^{59}$

A curto/ médio prazo é expectável o aparecimento no mercado de EPI e DM com maior potencial de inovação em termos não só de proteção, mas também de durabilidade, ergonomia e conforto. Também nos processos de conservação, limpeza e descontaminação se esperam desenvolvimentos com impacto positivo em termos ambientais.

\section{CONCLUSÃO}

Nas últimas décadas, tem-se testemunhado o surgimento de novos agentes infeciosos, alguns dos quais se tornaram grandes ameaças globais. A nova pandemia por SARS-CoV-2 é um exemplo de uma emergência de saúde pública de âmbito internacional, associada a consideráveis 
desafios sociais e económicos. As repercussões nos sistemas de saúde são avassaladoras, com um grande risco de colapso perante um aumento desmesurado da procura, a par de recursos humanos, de infraestrutura e logísticos finitos. A nível das unidades de saúde há o risco de que surtos nosocomiais sejam amplificadores locais importantes. Perante tal, práticas de controlo de infeção são de importância crítica no funcionamento destas unidades, nomeadamente: na identificação precoce e isolamento de casos suspeitos/confirmados; definição de circuitos distintos para estes doentes; implementação de medidas transversais para distanciamento físico; reforço da etiqueta respiratória e higiene das mãos, assim como descontaminação de superfícies e equipamentos. A disponibilidade de EPI, e formação adequada dos profissionais, é outro dos pilares básicos de atuação. A nível da proteção facial e respiratória existe, atualmente, fraca evidência de que a utilização de respiradores permita maior proteção que uma máscara cirúrgica, para o SARS-CoV-2 ou outros vírus (com exceção dos PGA's, em que a utilização de um respirador é recomendada). Por sua vez, a proteção ocular deve ser garantida sempre que houver risco de salpicos, gotículas ou aerossóis. ${ }^{22}$ Por fim, de referir que, perante a contingência internacional na disponibilidade de EPI, a indústria nacio-

\section{REFERÊNCIAS}

1. Bunyan D, Ritchie L, Jenkins D, Coia JE. Respiratory and facial protection: a critical review of recent literature. J Hosp Infect. 2013;85:165-9.

2. Coia JE, Ritchie L, Adisesh A, Makison Booth C, Bradley C, Bunyan D, et al. Guidance on the use of respiratory and facial protection equipment. J Hosp Infect. 2013;85:170-82.

3. ECDC. Event background COVID-19. Stockholm: European Center for Disease Control and Prevention; 2020.

4. Hawker J, Begg N, Reintjes R, Ekdahl, Edeghere O, Steenbergen, J. Communicable disease control and health protection handbook. $4^{\mathrm{a}} \mathrm{ed}$. Oxford: Blackwell Publishing; 2019.

5. Word Health Organization. WHO announces COVID-19 outbreak a pandemic. [consultado 2020 abr 2]. Disponível em: http://www.euro. who.int/en/health-topics/health-emergencies/coronavirus-covid-19/ news/news/2020/3/who-announces-covid-19-outbreak-a-pandemic.

6. European Centre for Disease Prevention and Control. Infection prevention and control for COVID-19 in healthcare settings - Second update. 31 March 2020. Stockholm: ECDC; 2020.

7. Weber DJ, Rutala WA, Fischer WA, Kanamori H, Sickbert-Bennett EE. Emerging infectious diseases: focus on infection control issues for novel coronaviruses (severe acute respiratory syndrome-CoV and Middle East respiratory syndrome-CoV), hemorrhagic fever viruses (Lassa and Ebola), and highly pathogenic avian influenza viruses, $A(H 5 N 1)$ and A(H7N9). Am J Infect Control. 2016;44:e91-100.

8. Wang P, Anderson N, Pan Y, Poon L, Charlton C, Zelyas N, et al. The SARS-CoV-2 outbreak: diagnosis, infection prevention, and public perception. Clin Chem. 2020 (in press). doi: 10.1093/clinchem/hvaa080.

9. Cheng VC, Chan JF, To KT, Yuen KY. Clinical management and infection control of SARS: lessons learned. Antiviral Res. 2013;100:407-19.

10. European Centre for Disease Prevention and Control. Coronavirus disease 2019 (COVID-19) in the EU/EEA and the UK - ninth update, 23 April 2020. Stockholm: ECDC; 2020.

11. Freitas G, Sá Machado R, editores. Plano Nacional de Preparação e Resposta à Doença pelo novo Coronavirus (COVID-19). Lisboa: Direção-Geral da Saúde; 2020.

12. European Centre for Disease Prevention and Control. Using face masks in the community. 8th April 2020. Stockholm: ECDC; 2020.

13. Siegel JD, Rhinehart E, Jackson M, Chiarello L; Health Care Infection Control Practices Advisory Committee. 2007 Guideline for Isolation nal tem respondido de forma célebre na sua produção, de modo a garantir a segurança de todos os seus utilizadores, revelando um sentido de responsabilidade social perante este desafio de proporções ainda não inteiramente conhecidas. A seguir a este desafio sanitário, outros surgirão, tal como alerta o virologista Nathan Wolfe, "(...) nos 100 anos desde que descobrimos os vírus, os seres humanos percorreram um longo caminho para entendê-los. Ainda resta muito trabalho...." ${ }^{60}$

\section{AGRADECIMENTOS}

Nesta pandemia por SARS-CoV-2, conscientes do grande esforço e risco a que estão sujeitos, deixamos um louvor à atuação e perseverança dos profissionais de saúde.

\section{CONFLITOS DE INTERESSE}

Os autores declaram não ter conflitos de interesses relacionados com o presente trabalho.

\section{FONTES DE FINANCIAMENTO}

Este trabalho não recebeu qualquer tipo de suporte financeiro de nenhuma entidade no domínio público ou privado.

Precautions: Preventing Transmission of Infectious Agents in Health Care Settings. Am J Infect Control. 2007;35:S65-164.

14. Autoridade Nacional do Medicamento e Produtos de Saúde. Máscaras cirúrgicas - DM versus aparelhos de proteção respiratória filtrantes EPI. Lisboa: INFARMED; 2020

15. Almeida T, Fernandes A, Marques E, Carneiro L, Carvalho M, Xavier V. Guia de seleção de aparelhos de proteção respiratória filtrantes. Lisboa: Autoridade para as Condições de Trabalho; 2016.

16. Atlanta Center for Disease Control and Prevention;. NIOSH approved particulate filtering facepiece respirators. 2020. [consultado $2020 \mathrm{abr}$ 2]. Disponível em: http://www.cdc.gov/niosh/.

17. Offeddu V, Yung CF, Low MS, Tam CC. Effectiveness of masks and respirators against respiratory infections in healthcare workers: a systematic review and meta-analysis. Clin Infect Dis. 2017;65:1934-42.

18. Radonovich LJ Jr, Simberkoff MS, Bessesen MT, Brown AC, Cummings DA, Gaydos CA, et al. N95 respirators vs medical masks for preventing influenza among health care personnel: a randomized clinical trial. JAMA. 2019;322:824-33.

19. Long Y, Hu T, Liu L, Chen R, Guo Q, Yang L, et al. Effectiveness of N95 respirators versus surgical masks against influenza: a systematic review and meta-analysis. J Evid Based Med. 2020;13:93-101.

20. Chu DK, Akl EA, Duda S, Solo K, Yaacoub S, Schünemann HJ, et al. Physical distancing, face masks, and eye protection to prevent personto-person transmission of SARS-CoV-2 and COVID-19: a systematic review and meta-analysis. Lancet. 2020 (in press). doi: 10.1016/S01406736(20)31142-9.

21. Comissão Europeia. Recomendação (UE) 2020/403 da Comissão Europeia de 13 de março de 2020 - Procedimentos de avaliação da conformidade e de fiscalização do mercado face à ameaça da COVID-19. [consultado 2020 abr 16]. Disponível em: https://eur-lex. europa.eu/eli/reco/2020/403/oj.

22. Decreto-Lei n. ${ }^{\circ}$ 14-E/2020. Diário da República, I Série, n. ${ }^{\circ} 72$ (2020/04/13). p. 86-(2-5).

23. Direção-Geral da Saúde. Norma n²9/2012 - Precauções Básicas em Controlo de Infeção. Lisboa: DGS; 2013.

24. Direção-Geral da Saúde. Informação nº 09/2020 - COVID-19: Fase de Mitigação - Uso de Máscaras na Comunidade. Lisboa: DGS; 2020.

25. European Centre for Disease Prevention and Control. Guidance for wearing and removing personal protective equipment in healthcare 
settings for the care of patients with suspected or confirmed COVID-19. Stockholm: ECDC; 2020.

26. World Health Organization. Advice on the use of masks in the context of COVID-19: interim guidance, 6 April 2020. Genebra: WHO; 2020.

27. Atlanta Center for Disease Control and Prevention. Infection Control Guidance for Healthcare Professionals about Coronavirus (COVID-19) - Using Personal Protective Equipment (PPE). 2020. [consultado 2020 abr 16]. Disponível em: http://www.cdc.gov/coronavirus/2019-ncov/hcp/ using-ppe.html.

28. European Centre for Disease Prevention and Control. Safe use of personal protective equipment in the treatment of infectious diseases of high consequence. Stockholm: ECDC; 2014.

29. Shaffer R, Cichowicz JK, Chew G, Hsu J. Non-occupational uses of respiratory protection - what public health organizations and users need to know. 2018. NIOSH. [consultado 2020 abr 17]. Disponível em: https:// blogs.cdc.gov/niosh-science-blog/2018/01/04/respirators-public-use/.

30. Direção Geral da Saúde. Norma nº 007/2020 de 29/03/2020 - Prevenção e Controlo de Infeção por SARS-CoV-2 (COVID-19): Equipamentos de Proteção Individual (EPI). Lisboa: DGS; 2020.

31. World Health Organization. Infection prevention and control during health care when COVID-19 is suspected. 19 March 2020. Genebra: WHO; 2020.

32. Atlanta Center for Disease Control and Prevention. Interim Infection Prevention and Control Recommendations for Patients with Suspected or Confirmed Coronavirus Disease 2019 (COVID-19) in Healthcare Settings. 2020. Atlanta: CDC; 2020.

33. Kampf G, Todt D, Pfaender S, Steinmann E. Persistence of coronaviruses on inanimate surfaces and their inactivation with biocidal agents. J Hosp Infect. 2020;104:246-51.

34. van Doremalen N, Bushmaker T, Morris DH, Holbrook MG, Gamble A, Williamson BN, et al. Aerosol and surface stability of SARS-CoV-2 as compared with SARS-CoV-1. N Engl J Med. 2020;382:1564-7.

35. Cook TM. Personal protective equipment during the coronavirus disease (COVID) 2019 pandemic - a narrative review. Anaesthesia. 2020;75:920-7

36. Liu Y, Ning Z, Chen Y, Guo M, Liu Y, Gali N, et al. Aerodynamic analysis of SARS-CoV-2 in two Wuhan hospitals. Nature. 2020 (in press). doi: 10.1038/s41586-020-2271-3.

37. Wong SC, Kwong RT, Wu TC, Chan JW, Chu MY, Lee SY, et al. Risk of nosocomial transmission of coronavirus disease 2019: an experience in a general ward setting in Hong Kong. J Hosp Infect. 2020;105:119-27.

38. Rubens JH, Karakousis PC, Jain SK. Stability and viability of SARSCoV-2. N Engl J Med. 2020;382:1962-3.

39. Verbeek JH, ljaz S, Mischke C, Ruotsalainen JH, Mäkelä E, Neuvonen $\mathrm{K}$, et al. Personal protective equipment for preventing highly infectious diseases due to exposure to contaminated body fluids in healthcare staff. Cochrane Database Syst Rev. 2016;4:CD011621.

40. Livingston E, Desai A, Berkwits M. Sourcing personal protective equipment during the COVID-19 Pandemic. JAMA. 2020 (in press). doi: 10.1001/jama.2020.5317.

41. World Health Organization. Rational use of personal protective equipment for coronavirus disease (COVID-19) and considerations during severe shortages: interim guidance. 6 April 2020. Genebra: WHO; 2020.

42. European Centre for Disease Prevention and Control. Personal protective equipment (PPE) needs in healthcare settings for the care of patients with suspected or confirmed 2019-nCoV. Stockholm:ECDC; 2020.

43. Ferioli M, Cisternino C, Leo V, Pisani, Palange P, Nava S. Protecting healthcare workers from SARS-CoV-2 infection: practical indications. Eur Respir Rev. 2020;29:200068.
44. Public Health England. Guidance - COVID-19 personal protective equipment (PPE) - $3^{\text {rd }}$ May 2020. London: PHE; 2020.

45. Tran K, Cimon K, Severn M, Pessoa-Silva CL, Conly J. Aeroso generating procedures and risk of transmission of acute respiratory infections to healthcare workers: a systematic review. PLoS ONE. 2012; $7:$ e35797.

46. Judson S, Munster V. Nosocomial transmission of emerging viruses via aerosol-generating medical procedures. Viruses. 2019;11:940.

47. Health Protection Surveillance Centre. Use of PPE to support infection prevention and control practice when performing aerosol generating procedures on confirmed or clinically suspected covid-19 cases in a pandemic situation. Dublin: HPSC; 2020.

48. Liu W, Tang F, Fang LQ, De Vlas SJ, Ma HJ, Zhou JP, et al. Risk factors for SARS infection among hospital healthcare workers in Beijing: a case control study. Trop Med Int Health. 2009;14:52-9.

49. Wax RS, Christian MD. Practical recommendations for critical care and anesthesiology teams caring for novel coronavirus (2019-nCoV) patients. Can J Anaesth. 2020;67:568-76.

50. Thompson KA, Pappachan JV, Bennett AM, Mittal H, Macken S, Dove $\mathrm{BK}$, et al. Influenza aerosols in UK hospitals during the H1N1 (2009) pandemic-the risk of aerosol generation during medical procedures. PloS one 2013;8:e56278.

51. Wahidi MM, Shojaee S, Lamb CR, Ost D, Maldonado F, Eapen G, et al. The use of bronchoscopy during the COVID-19 pandemic: CHEST/ AABIP Guideline and Expert Panel Report. Chest. 2020 (in press). doi: 10.1016/j.chest.2020.04.036

52. Bugalho A, Guedes F, Novais Bastos H, Mota L, Vaz Rodrigues L, Boléo-Tomé JP. Documento de posição da Sociedade Portuguesa de Pneumologia para a realização de broncoscopias durante o surto de COVID-19. Lisboa: Sociedade Portuguesa de Pneumologia; 2020.

53. Fowler RA, Guest CB, Lapinsky SE, Sibbald WJ, Louie M, Tang P, et al. Transmission of severe acute respiratory syndrome during intubation and mechanical ventilation. Am J Respir Crit Care Med. 2004;169:1198202.

54. Guan L, Zhou L, Zhang J, Peng W, Chen R. More awareness is needed for severe acute respiratory syndrome coronavirus 2019 transmission through exhaled air during non-invasive respiratory support: experience from China. Eur Respir J. 2020;55:2000352.

55. Ng K, Poon BH, Kiat Puar TH, Quah J, Loh WJ, Wong YJ, et al COVID-19 and the risk for health care workers - a case report. Ann Intern Med. 2020;172:766-7.

56. Canadian Society of Otolaryngology-Head \& Neck Surgery Executive Committee. Guidance for Health Care Workers Performing Aerosol Generating Medical Procedures during the COVID-19 Pandemic. 2020. [consultado 2020 abr 18]. Disponível em: http://www.entcanada.org/wpcontent/uploads/Protocol-for-COVID-and-AGMP-3-iw-mailer.pdf.

57. Intercollegiate General Surgery Group. Updated General Surgery Guidance on COVID-19. 6 April 2020.. [consultado 2020 abr 18]. Disponível em: http://www.rcseng.ac.uk/coronavirus/joint-guidance-forsurgeons-v2/.

58. Autoridade Nacional do Medicamento e Produtos de Saúde, Direção Geral da Saúde, Autoridade de Segurança Alimentar e Económica, Instituto Português da Qualidade. Máscaras destinadas à utilização no âmbito da COVID-19 - Especificações Técnicas. Lisboa: INFARMED, DGS, ASAE e IPQ; 2020.

59. Centro Tecnológico Têxtil e do Vestuário de Portugal. Máscaras COVID-19 Aprovado. 2020. [consultado 2020 abr 16]. Disponível em: http://www.citeve.pt/artigo/selo_mascara_aprovado.

60. Wolfe N. The viral storm - the dawn of a new pandemic Age. London: Penguin Publisher; 2013. 